\title{
ARBITRARY ORDER NODAL MIMETIC DISCRETIZATIONS OF ELLIPTIC PROBLEMS ON POLYGONAL MESHES WITH ARBITRARY REGULAR SOLUTION
}

\author{
L. Beirão da Veiga ${ }^{1}$ and G. Manzini ${ }^{2}$ \\ ${ }^{1}$ Department of Mathematics "F. Enriques" at the University of Milan, Italy \\ ${ }^{2}$ Los Alamos National Laboratory, Theoretical Division, MS B284, Los Alamos, NM, 87545 \\ (gmanzini@lanl.gov)
}

\begin{abstract}
We present a new family of mimetic methods on unstructured polygonal meshes for the diffusion problem in primal form for solution with regularity $C^{\alpha}(\Omega)$ for any integer $\alpha \geq 0$. These methods are derived from a local consistency condition that is exact for polynomials of degree $m=\alpha+1$. The degrees of freedom are (a) solution and derivative values of various degree at the mesh vertices and $(b)$ solution moments inside polygons. Theoretical results concerning the convergence of the method are briefly summarized and an optimal error estimate is given in a mesh-dependent norm that mimics the energy norm. Numerical experiments confirm the convergence rate that is expected from the theory.
\end{abstract}

Keywords: Diffusion problem, mimetic finite difference method, polygonal mesh, generalized mesh, high-order scheme

\section{INTRODUCTION}

The mimetic discretizations have been developed since the early eighties to incorporate important properties of physical and mathematical models into the discrete framework. Such discretizations in mixed or mixed-hybrid form [14] and its earlier version, the supportoperator method [21,27] has been successfully employed for solving problems of electromagnetism [22] gas dynamics [16] linear diffusion (see, e.g.,[7,11,15,18,20,24,26], and references therein), convection-diffusion [4,19], Stokes [5,6,8], elasticity [3], Reissner-Mindlin plates [12], eigenvalues [17], two-phase flows in porous media [1,25]. A posteriori estimators were developed in [2,10] and monotonicity criteria were investigated in [23]. Here, we consider the possibility of building a discrete method that incorporates a given degree $\alpha \in \mathbb{N}$ of $C^{\alpha}$ global regularity into the discrete solution. In the conforming finite element method, the discrete spaces used are globally $C^{0}$ and the construction of finite element methods with a regularity that is higher than one is prohibitive. In the mimetic approach, we can build highly regular methods that are associated to a discrete space with arbitrary $C^{\alpha}$ regularity. To this end, we consider a mimetic construction that depends on two integer parameters, the polynomial degree $m$ and the regularity index $\alpha$. The parameter $m$ determines the degree of the polynomials that satisfy the consistency condition and therefore the $O\left(h^{m}\right)$ order of convergence of the method, provided the solution is sufficiently regular. The parameter $\alpha$ determines the global smoothness of the underlying discrete space, that is of $C^{\alpha}$ regularity across the edges of the mesh. Although, in principle, we can formulate schemes with $m \geq \alpha+1$, we will concentrate to methods satisfying the minimal condition $m=\alpha+1$. 
The outline of the paper is as follows. In Section 2 we introduce the mathematical model and we present the formulation of the nodal mimetic finite difference method. In Section 3 we briefly summarize the regularity assumptions on the mesh and the convergence result. In Section 4 we illustrate the behavior of the method in solving a representative test case. In Section 5 we offer final remarks and conclusions.

\section{The mathematical model and its mimetic formulation}

Let us consider the steady diffusion problem for the scalar solution field $u$ given by

$$
\begin{aligned}
\operatorname{div}(\mathrm{K} \nabla u)=f & \text { in } \Omega, \\
u=g & \text { on } \Gamma,
\end{aligned}
$$

where $\Omega \subset \mathbb{R}^{2}$ is the computational domain, $\Gamma$ is the boundary of $\Omega, \mathrm{K}$ is the diffusion tensor describing the material properties, $f$ is the forcing term and $g$ the boundary function that defines the non-homogeneous Dirichlet boundary condition.

Let us consider the functional space $H_{g}^{1}(\Omega)=\left\{v \in H^{1}(\Omega), v_{\mid \Gamma}=g\right\}$. Problem (1)-(2) can be restated in the variational form:

find $u \in H_{g}^{1}(\Omega)$ such that

$$
\int_{\Omega} \mathrm{K} \nabla u \cdot \nabla v d V=\int_{\Omega} f v d V \quad \forall v \in H_{0}^{1}(\Omega) .
$$

The existence and uniqueness of the weak solution follows from continuity and coercivity of the bilinear form in (3) uner suitable regularity assumptions on $\mathrm{K}$ and the domain $\Omega$.

We approximate (3) on a sequence of meshes $\left\{\Omega_{h}\right\}_{h}$ that partition the domain $\Omega$; the meshes are labeled by $h$, which is the mesh size and is defined by $h=\max _{\mathrm{P} \in \Omega_{h}} h_{\mathrm{P}}$ where $h_{\mathrm{P}}=\sup _{\mathbf{x}, \mathbf{y} \in \mathrm{P}}|\mathbf{x}-\mathbf{y}|$ is the diameter of the polygonal cell $\mathrm{P} \in \Omega_{h}$.

On each mesh $\Omega_{h}$, we approximate the functional space $H^{1}(\Omega) \cap C^{0}(\bar{\Omega})$ by the linear space $\mathcal{V}_{h}$, a collection of discrete scalar fields, also referred to as grid functions. Grid functions are properly defined through the degrees of freedom, which can be interpreted as nodal values of functions and derivatives of functions in the continuum setting, and integral moment associated with the mesh cells, cf. the definitions given in subsection 2.3. Then, we introduce the bilinear form $\mathcal{A}_{h}(\cdot, \cdot): \mathcal{V}_{h} \times \mathcal{V}_{h} \rightarrow \mathbb{R}$ that approximates the left-hand side of (3) and the bilinear form $(\cdot, \cdot)_{h}: L^{2}(\Omega) \times \mathcal{V}_{h} \rightarrow \mathbb{R}$ that approximates the right-hand side of (3), i.e.,

$$
\mathcal{A}_{h}\left(u^{\mathrm{I}}, v^{\mathrm{I}}\right) \approx \int_{\Omega} \mathrm{K} \nabla u \cdot \nabla v d V, \quad\left(f, v^{\mathrm{I}}\right)_{h} \approx \int_{\Omega} f v d V
$$

In the nodal mimetic finite difference formulation, the Dirichlet boundary conditions are essential and are incorporated through the subspace $\mathcal{V}_{h, g}$ of $\mathcal{V}_{h}$. The space $\mathcal{V}_{h, g}$ is formed by the discrete scalar fields of $\mathcal{V}_{h}$ whose degrees of freedom associated with the boundary edges approximate the boundary datum $g$. Finally, the mimetic finite difference method for (3) reads:

Find $u_{h} \in \mathcal{V}_{h, g}$ such that:

$$
\mathcal{A}_{h}\left(u_{h}, v_{h}\right)=\left(f, v_{h}\right)_{h} \quad \forall v_{h} \in \mathcal{V}_{h, 0}
$$

The well-posedness of the numerical approximation (4) follows from the coercivity and the continuity properties of the bilinear form $\mathcal{A}_{h}(\cdot, \cdot)$. 


\subsection{Notation}

We denote a generic mesh vertex by $v$ and its coordinate vector by $x_{v}$, a generic mesh edge by $f$ and its length by $|f|$, the area of polygon $P$ by $|P|$, and its boundary by $\partial P$. The orientation of each mesh edge $f$ is reflected by its unit normal vector $\mathbf{n}_{f}$, which is fixed once and for all. For any polygon $P$ and any edge $f$ of $\partial P$, we define the unit normal vector $\mathbf{n}_{P, f}$ that points out of $\mathrm{P}$. Clearly, the sign $s_{\mathrm{P}, \mathrm{f}}=\mathbf{n}_{\mathrm{f}} \cdot \mathbf{n}_{\mathrm{P}, \mathrm{f}}$ may be either +1 or -1 depending on the mutual orientation of $f$ and $\partial P$. We denote the set of the mesh vertices by $\mathcal{V}$. In the following we assume that the tensor $\mathrm{K}$ is constant.

\subsection{The mimetic bilinear form $\mathcal{A}_{h, \mathrm{P}}$}

The mimetic bilinear form $\mathcal{A}_{h}$ is given by the assembly of the local bilinear forms $\mathcal{A}_{h, \mathrm{P}}: H^{1}(\mathrm{P}) \times H^{1}(\mathrm{P}) \rightarrow \mathbb{R}$, which are defined for each polygonal cell $\mathrm{P}$. Each bilinear form $\mathcal{A}_{h, \mathrm{P}}$ is an approximation of the integral

$$
\mathcal{A}_{\mathrm{P}}(u, v)=\int_{\mathrm{P}} \nabla u \cdot \mathrm{K} \nabla v d V, \quad u, v \in H^{1}(\mathrm{P}) .
$$

Such approximation is based on the degrees of freedom of $u$ and $v$ and satisfy an exactness property that involves the functional space $\mathbb{P}_{m}(\mathrm{P})$ of the polynomials of degree up to $m$ and the functional space

$$
V^{m}=\left\{v \in H^{1}(\mathrm{P}) \cap C^{\alpha}(\mathrm{P}) \text { such that } v_{\mid \mathrm{f}} \in \mathbb{P}_{m}(\mathrm{f}) \quad \forall \mathrm{f} \in \partial \mathrm{P}\right\} .
$$

The mimetic bilinear form $\mathcal{A}_{h, \mathrm{P}}$ is such that:

(i) it is symmetric with respect to its two arguments;

(ii) it satisfies the exactness condition:

$$
\mathcal{A}_{h, \mathrm{P}}\left(p^{\mathrm{I}}, v^{\mathrm{I}}\right)=\mathcal{A}_{\mathrm{P}}(p, v) \quad \text { for all } p \in \mathbb{P}_{m}(\mathrm{P}), v \in V^{m},
$$

where $p^{\mathrm{I}}$ and $v^{\mathrm{I}}$ are denote the interpolation of $p$ and $v$ with respect to the degrees of freedom of $\mathcal{V}_{h, \mathrm{P}}$.

Let us further develop equation (6). We integrate by parts the right-hand side of equation (5) and we obtain:

$$
\mathcal{A}_{\mathrm{P}}(p, v)=-\int_{\mathrm{P}} \operatorname{div}(\mathrm{K} \nabla p) v d V+\sum_{\mathrm{f} \in \partial \mathrm{P}} \int_{\mathrm{f}}\left(\mathbf{n}_{\mathrm{P}, \mathrm{f}} \cdot \mathrm{K} \nabla p\right) v d S .
$$

As $p$ is a polynomial of degree $m$ and $\mathrm{K}$ is a constant tensor field on $\mathrm{P}$, the divergence term, i.e., $\operatorname{div}(\mathrm{K} \nabla p)$ is a polynomial of degree $(m-2)$. Let us consider the basis $\left\{\varphi_{k, i}\right\}_{k=0, \ldots, m-2, i=0, \ldots, k}$ of the polynomial space $\mathbb{P}_{m}(\mathrm{P})$. This basis collects $(m-1)$ sets of polynomials of degree $k=0, \ldots, m-2$, i.e., $\left\{\varphi_{k, i}\right\}_{i=0, \ldots, k}$, each one of which being a basis of the set of polynomials of degree exactly equal to $k$. We express $\operatorname{div}(\mathrm{K} \nabla p)$ as a linear combination of these basis functions:

$$
\operatorname{div}(\mathrm{K} \nabla p)=\sum_{k=0}^{m-2} \sum_{i=0}^{k} \alpha_{k, i}(p) \varphi_{k, i}
$$


where the " $\alpha$ " coefficients obviously depend on $p$. Moreover, since $v$ belongs to $V^{m}$, its the trace $v_{\mid \mathrm{f}}$ along the edge $\mathrm{f}$ of $\partial \mathrm{P}$ is also a polynomial of degree $m$. Substituting (8) in (7) we reformulate equation (6) as

$$
\mathcal{A}_{h, \mathrm{P}}\left(p^{\mathrm{I}}, v^{\mathrm{I}}\right)=\mathcal{I}_{\mathrm{P}}\left(v^{\mathrm{I}}, p\right)+\sum_{\mathrm{f} \in \partial \mathrm{P}} \mathcal{I}_{\mathrm{f}}\left(v^{\mathrm{I}}, p\right)
$$

by introducing the surface integral

$$
\mathcal{I}_{\mathrm{P}}\left(v^{\mathrm{I}}, p\right)=-\sum_{k=0}^{m-2} \sum_{i=0}^{k} \alpha_{k, i}(p) \int_{\mathrm{P}} v \varphi_{k, i} d V,
$$

and the edge integrals associated with each edge $f \in \partial P$

$$
\mathcal{I}_{\mathrm{f}}\left(v^{\mathrm{I}}, p\right)=\int_{\mathrm{f}} v_{\mid \mathrm{f}} \mathbf{n}_{\mathrm{P}, \mathrm{f}} \cdot \mathrm{K} \nabla p d S
$$

and both definitions use the degrees of freedom $v^{\mathrm{I}}$ of function $v$. The surface integral in (10) is expressed in terms of $v^{\mathrm{I}}$ if we define the set of internal degrees of freedom of $v$ as the $m(m-1) / 2$ integral moments:

$$
v_{\mathrm{P}, k, i}=\frac{1}{|\mathrm{P}|} \int_{\mathrm{P}} v \varphi_{k, i} d V, \quad k=0, \ldots, m-2, \quad i=0,1, \ldots, k .
$$

Likewise, we can express the edge integral (11) in terms of the degrees of freedom of the method if we properly define a set of nodal degrees of freedom along each edge $f$. To such purpose, we consider the value of $v$ and of its derivatives at the vertices of $\mathrm{f}$ so that the trace $v_{\mid f}$ is the unique solution of a polynomial interpolation problem using such values as input data. In subsection 2.3 we discuss how to select the nodal degrees of freedom.

Finally, we require that $\mathcal{A}_{h, \mathrm{P}}$ is symmetric bilinear form that satisfies a stability condition that guarantees the correct scaling and kernel and a consistency condition that extends relation (9) to every mimetic field $v_{h}$.

(S1) spectral stability: there exists two positive constants $\sigma_{*}$ and $\sigma^{*}$ such that for every $v_{h, \mathrm{P}} \in \mathcal{V}_{h, \mathrm{P}}$ there holds:

$$
\sigma_{*}\left\|v_{h, \mathrm{P}}\right\|_{1, h, \mathrm{P}}^{2} \leq \mathcal{A}_{h, \mathrm{P}}\left(v_{h, \mathrm{P}}, v_{h, \mathrm{P}}\right) \leq \sigma^{*}\left\|v_{h, \mathrm{P}}\right\|_{1, h, \mathrm{P}}^{2}
$$

(S2) local consistency: for every $v_{h, \mathrm{P}} \in \mathcal{V}_{h, \mathrm{P}}$ and for every $p \in \mathbb{P}_{m}(\mathrm{P})$ there holds:

$$
\mathcal{A}_{h, \mathrm{P}}\left(v_{h, \mathrm{P}}, p_{\mathrm{P}}^{\mathrm{I}}\right)=\mathcal{I}_{\mathrm{P}}\left(v_{h, \mathrm{P}}, p\right)+\sum_{\mathrm{f} \in \partial \mathrm{P}} \mathcal{I}_{\mathrm{f}}\left(v_{h, \mathrm{P}}, p\right) .
$$

Property (S1) combined with the boundary conditions implies that the bilinear form $\mathcal{A}_{h}$ is coercive, thus ensuring the existence of a unique solution to the discrete problem (4). On its turn, (S2) implies $\mathcal{A}_{h, \mathrm{P}}\left(v^{\mathrm{I}}, p^{\mathrm{I}}\right)$ coincides with $\mathcal{A}_{\mathrm{P}}(v, p)$ when both $v$ and $p$ are polynomials of degree less than or equal to $m$. 

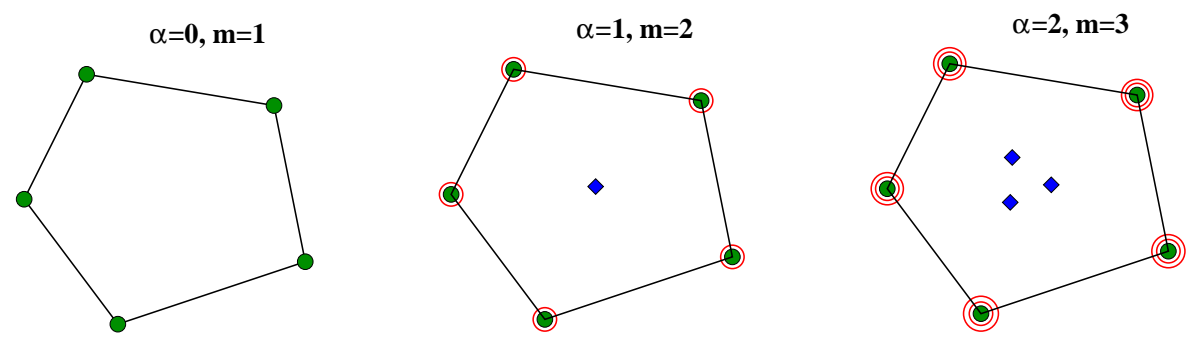

Figure 1. Degrees of freedom for $(\alpha, m)=(0,1),=(1,2),=(2,3)$. The symbols shown in the plots represent vertex values (dot), vertex first-order derivatives (one circle), vertex first- and second-order derivatives (two circles), and polynomial moments (diamonds in the interior).

Table 1. Edge degrees of freedom for $\alpha=0,1,2$ and $m=\alpha+1$.

\begin{tabular}{|c|c|c|c|}
\hline$m$ & dofs & conditions & interp. space \\
\hline 1 & $\longrightarrow$ & $2 \mathrm{v}$ & $\mathbb{P}_{1}(\mathrm{f})$ \\
\hline 2 & () & $2 v+2 v^{\prime}$ & $\mathbb{P}_{3}(\mathrm{f})$ \\
\hline 3 & (a) (a) & $2 \mathrm{v}+2 \mathrm{v}^{\prime}+2 \mathrm{v}^{\prime \prime}$ & $\mathbb{P}_{5}(\mathrm{f})$ \\
\hline
\end{tabular}

\subsection{Nodal degrees of freedom}

In Figure 1 we depict the first possible choices of the nodal degrees of freedom on a pentagonal element. We denote the nodal degrees of freedom associated with the vertex $\mathrm{v}$ through the general notation $v_{\mathrm{v}, k, i}$ where the subindex $k=0, \ldots, \alpha$ is the order of the partial derivative and $i=0, \ldots, k$ indicates the partial derivative with respect to $x^{k-i} y^{i}$, i.e., $\partial v\left(\mathbf{x}_{\mathrm{v}}\right) /$ $\partial x^{k-i} y^{i}$. The first (and simplest) choices of nodal degrees of freedom ar illustrated in the leftmost columns of Table 1. In this table, column "dofs" shows the number and kind of degrees of freedom for the pair $(\alpha, m)$ that is available at edge $\mathrm{f}$; the symbols are the same used in Figure 1. Column "conditions" shows the type and number of conditions available to build such polynomial interpolation; $\mathrm{v}$ denotes solution values at vertices, $\mathrm{v}^{\prime}=\left(\mathrm{v}_{x}, \mathrm{v}_{y}\right)$ denotes the gradient at the vertices, $\mathrm{v}^{\prime \prime}=\left(\mathrm{v}_{x x}, \mathrm{v}_{x y}, \mathrm{v}_{y y}\right)$ denotes the hessian components at the vertices. Column "interp. space" show the interpolation space that can be built using the information available in the previous column. In accordance with the symbols used in Figure 1, the "dots" indicate point evaluation of a given function and the concentric circles indicate the point evaluation of the function derivatives. We use the degrees of freedom $v_{\mathrm{f}}$ associated with edge $\mathrm{f}$ to define an interpolation problem that returns the polynomial of maximum degree that can be determined using $v_{\mathrm{f}}$. To determine $v_{\mathrm{f}}$ we impose that the interpolant reproduces the value of $v$ and of the tangential derivatives of $v$ along $\mathrm{f}$ of order up to $\alpha$ (when $\alpha>0$ ) at the edge vertices $v^{\prime}$ and $v^{\prime \prime}$. Hence, we impose $(\alpha+1)$ conditions at each vertex and the degree of the interpolant must be equal to $(2 \alpha+1)$.

Let $s \in[0,|\mathrm{f}|]$ be a local coordinate defined on edge $\mathrm{f}$ and such that $s=0$ corresponds to $\mathrm{v}^{\prime}$ and $s=|\mathrm{f}|$ to $\mathrm{v}^{\prime \prime}$. The three simplest situations that we discuss below are illustrated in Table 1. 
- For $\alpha=0$, we build the unique linear interpolant that satisfies the two conditions on the function values at the vertices of $f$ :

$$
v_{\mathrm{f}}(0)=v_{\mathrm{v}^{\prime}}, v_{\mathrm{f}}(|\mathrm{f}|)=v_{\mathrm{v}^{\prime \prime}} .
$$

- For $\alpha=1$, we build the unique cubic interpolant that satisfies the four conditions on the function values and the first derivatives at the vertices of $\mathrm{f}$, these latters being denoted by $v_{\mathrm{v}},\left(v_{\mathrm{v}, x}, v_{\mathrm{v}, y}\right)$ for $\mathrm{v}=\in\left\{\mathrm{v}^{\prime}, \mathrm{v}^{\prime \prime}\right\}$ :

$$
\begin{aligned}
v_{\mathrm{f}}(0) & =v_{\mathrm{v}^{\prime}} \\
v_{\mathrm{f}}(|\mathbf{f}|) & =v_{\mathrm{v}^{\prime \prime}} \\
v_{\mathrm{f}}^{\prime}(0) & =\mathbf{t}_{\mathrm{f}} \cdot\left(v_{\mathrm{v}^{\prime}, x}, v_{\mathrm{v}^{\prime}, y}\right), \\
v_{\mathrm{f}}^{\prime}(|\mathbf{f}|) & =\mathbf{t}_{\mathbf{f}} \cdot\left(v_{\mathrm{v}^{\prime \prime}, x}, v_{\mathrm{v}^{\prime \prime}, y}\right) .
\end{aligned}
$$

- For $\alpha=2$, we build the 5-degree interpolant that satisfies the six conditions on the function values and the first and second derivatives at each vertex, these latters being denoted by $v_{\mathrm{v}},\left(v_{\mathrm{v}, x}, v_{\mathrm{v}, y}\right),\left(v_{\mathrm{v}, x x}, v_{\mathrm{v}, x y}, v_{\mathrm{v}, y y}\right)$ for $\mathrm{v} \in\left\{\mathrm{v}^{\prime}, \mathrm{v}^{\prime \prime}\right\}$ :

$$
\begin{aligned}
v_{\mathrm{f}}(0) & =v_{\mathrm{v}^{\prime}}, \\
v_{\mathrm{f}}(|\mathbf{f}|) & =v_{\mathrm{v}^{\prime \prime}} \\
v_{\mathrm{f}}^{\prime}(0) & =\mathbf{t}_{\mathrm{f}} \cdot\left(v_{\mathrm{v}^{\prime}, x}, v_{\mathrm{v}^{\prime}, y}\right), \\
v_{\mathrm{f}}^{\prime}(|\mathbf{f}|) & =\mathbf{t}_{\mathrm{f}} \cdot\left(v_{\mathrm{v}^{\prime \prime}, x}, v_{\mathrm{v}^{\prime \prime}, y}\right), \\
v_{\mathrm{f}}^{\prime \prime}(0) & =H\left(v_{\mathrm{v}^{\prime}, x x}, v_{\mathrm{v}^{\prime}, x y}, v_{\mathrm{v}^{\prime}, y y}\right)\left(\mathbf{t}_{\mathrm{f}}, \mathbf{t}_{\mathrm{f}}\right), \\
v_{\mathrm{f}}^{\prime \prime}(|\mathbf{f}|) & =H\left(v_{\mathrm{v}^{\prime \prime}, x x}, v_{\mathrm{v}^{\prime \prime}, x y}, v_{\mathrm{v}^{\prime \prime}, y y}\right)\left(\mathbf{t}_{\mathbf{f}}, \mathbf{t}_{\mathbf{f}}\right),
\end{aligned}
$$

where $H\left(v_{\mathrm{v}, x x}, v_{\mathrm{v}, x y}, v_{\mathrm{v}, y y}\right)\left(\mathbf{t}_{\mathbf{f}}, \mathbf{t}_{\mathrm{f}}\right)$ stands for the hessian matrix built using the second derivatives $v_{\mathrm{v}, x x}, v_{\mathrm{v}, x y}$ and $v_{\mathrm{v}, y y}$ at $\mathrm{v} \in\left\{\mathrm{v}^{\prime}, \mathrm{v}^{\prime \prime}\right\}$ and applied twice to the vector $\mathbf{t}_{\mathrm{f}}$ oriented from $\mathrm{v}^{\prime}$ to $\mathrm{v}^{\prime \prime}$.

In view of the previous definitions, we introduce a generic element of the mimetic space $\mathcal{V}_{h}$ as the collection of values

$$
v_{h}=\left\{\left(v_{\mathrm{v}, k, i}\right)_{\mathrm{v} \in \mathcal{V}, k=0, \ldots, \alpha, i=0, \ldots, k},\left(v_{\mathrm{P}, k, i}\right)_{\mathrm{P} \in \Omega_{h}, k=0, \ldots, m-2, i=0, \ldots, k}\right\},
$$

where $v_{\mathrm{P}, k, i}$ are the internal degres of freedom introduced in (12), and we define the linear space $\mathcal{V}_{h}$ as the collection of all such elements (with the obvious definitions of the sum of two of its elements and of the multiplication of an element of the space by a scalar). We also define $\mathcal{V}_{h, 0} \subset \mathcal{V}_{h}$ as the subspace satisfying homogeneous Dirichlet boundary conditions

$$
\mathcal{V}_{h, 0}=\left\{v_{h} \in \mathcal{V}_{h}: v_{\mathrm{v}, 0,0}=0 \forall \mathrm{v} \in \partial \Omega, v_{\mathrm{f}, 0, i}=0 \forall \mathrm{f} \in \partial \Omega, i=1, \ldots, \mathcal{N}_{k}\right\}
$$

\subsection{Discretization of the load term $(\cdot, \cdot)_{h}$}

Let $\mathcal{P}^{k}: L^{2}(\mathrm{P}) \rightarrow \mathbb{P}_{k}(\mathrm{P})$ be the $L^{2}$-orthogonal projector of scalar functions onto the space of polynomials of degree at most $k$. We denote the projection of $f$, the forcing term in the right-hand side of (3), on $\mathbb{P}_{m-2}(\mathrm{P})$ by $\hat{f}_{\mathrm{P}}$, i.e., $\hat{f}_{\mathrm{P}}=\mathcal{P}^{m-2}(f)$. Since $\hat{f}_{\mathrm{P}} \in \mathbb{P}_{m-2}(\mathrm{P})$, we reformulate it as a linear combination of the basis functions $\varphi_{k, i}$ :

$$
\hat{f}_{\mathrm{P}}=\sum_{k=0}^{m-2} \sum_{i=0}^{k} c_{k, i} \varphi_{k, i}
$$


using the $(m+1)(m+2) / 2$ real coefficients $c_{k, i}$. Then, we define

$$
\left(f, v_{h}\right)_{h}=\sum_{\mathrm{P} \in \Omega_{h}}\left[\hat{f}_{\mathrm{P}}, v_{h, \mathrm{P}}\right]_{\mathrm{P}}, \quad\left[\hat{f}_{\mathrm{P}}, v_{h, \mathrm{P}}\right]_{\mathrm{P}}=|\mathrm{P}| \sum_{k=0}^{m-2} \sum_{i=0}^{k} c_{k, i} v_{\mathrm{P}, k, i},
$$

where we use the coefficients $c_{k, i}$ from (15).

Remark 2.1. For any $v \in L^{2}(\mathrm{P})$ and any loading term $f \in L^{2}(\Omega)$ it holds that

$$
\left[\hat{f}_{\mathrm{P}}, v_{\mathrm{P}}^{I}\right]_{\mathrm{P}}=\int_{\mathrm{P}} \mathcal{P}^{m-2}(f) \mathcal{P}^{m-2}(v) d V=\int_{\mathrm{P}} \hat{f}_{\mathrm{P}} v d V \quad \forall \mathrm{P} \in \Omega_{h} .
$$

We recall that $v_{\mathrm{P}}^{I}=\left(v_{\mid \mathrm{P}}\right)^{I}$, and we note that (16) involves only the internal degrees of freedom of the interpolant $v^{I}$.

\subsection{Mesh-dependent norms}

Let $\bar{v}_{h, \mathrm{P}}$ denote the arithmetic mean of the vertex values of $v_{h, \mathrm{P}}$

$$
\bar{v}_{h, \mathrm{P}}=\frac{1}{N_{\mathrm{P}}^{\mathcal{V}}} \sum_{\mathrm{v} \in \partial \mathrm{P}} v_{\mathrm{v}} .
$$

Now, we introduce the following mesh-dependent norm for the elements of $\mathcal{V}_{h}$ that includes higher order terms but mimics the $H^{1}(\Omega)$ seminorm:

$$
\left\|v_{h}\right\|_{1, h}^{2}=\sum_{\mathbf{P} \in \Omega_{h}}\left\|v_{h}\right\|_{1, h, \mathrm{P}}^{2}
$$

where

$$
\begin{aligned}
\left\|v_{h}\right\|_{1, h, \mathrm{P}}^{2} & =\sum_{\mathrm{f} \in \partial \mathrm{P}} h_{\mathrm{P}}\left\|\frac{d v_{\mathrm{f}}}{d s}\right\|_{L^{2}(\mathrm{f})}^{2}+\sum_{k=1}^{\alpha} \sum_{\mathrm{f} \in \partial \mathrm{P}} h_{\mathrm{P}}^{2 k-1}\left\|\partial_{n}^{k} v_{\mathrm{f}}\right\|_{L^{2}(\mathrm{f})}^{2} \\
& +\left(v_{\mathrm{P}, 0,0}-\bar{v}_{h, \mathrm{P}}\right)^{2}+\sum_{k=1}^{m-2} \sum_{i=0}^{k}\left|v_{\mathrm{P}, k, i}\right|^{2} .
\end{aligned}
$$

Above, the symbol $v_{\mathrm{f}}$ denotes the reconstructed polynomial on the edge $f$ associated with the vertex date $v_{h}$, as described in the previous paragraph. The symbol $\frac{\partial v_{f}}{\partial s}$ indicates the tangential derivative of $v_{\mathrm{f}}$, while $\partial_{n}^{k} v_{\mathrm{f}}$ indicates the reconstruction of the normal derivatives of order $k$ for $1 \leq k \leq \alpha$. This reconstruction is analogous to the one that is made for the definition of the mimetic bilinear form. In fact, these polynomials are determined uniquely by imposing that they reproduce all the partial derivatives at $v^{\prime}$ and $v^{\prime \prime}$ that can be constructed using all the available data at the vertices. It turns out that for $k=\alpha$, i.e., the normal derivative with maximum order, we only have two conditions to impose, and the interpolation is always linear. Instead, for $1<k<\alpha$ (when $\alpha>1$ ), we can impose more conditions using also the tangential derivatives of $\partial_{n}^{k} v_{\mathrm{f}}(s)$ along the edge $\mathrm{f}$ and evaluated in $\mathrm{v}^{\prime}$ and $\mathrm{v}^{\prime \prime}$, and the degree of the interpolating polynomial is greater than one.

Note that the seminorms in (19) have a non trivial kernel that represents the constant functions on P. More precisely, it holds $\left\|v_{h}\right\|_{1, h, \mathrm{P}}=0$ if and only if it exists a constant $C \in \mathbb{R}$ such that

$$
v_{\mathrm{P}, 0,0}=\bar{v}_{h, \mathrm{P}}=C, \quad v_{\mathrm{v}, 0,0}=C \forall \mathrm{v} \in \partial \mathrm{P},
$$


and the remaining degrees of freedom vanish. This is equivalent to say that $\left\|v_{h}\right\|_{1, h, \mathrm{P}}=0$ if and only if $\left.v_{h}\right|_{\mathrm{P}}=C_{\mathrm{P}}^{\mathrm{I}}$ for some constant $C \in \mathbb{R}$. As a consequence, the global seminorm $\left\|v_{h}\right\|_{1, h}=0$ if and only if $v_{h}=C^{\text {I }}$ for some constant $C \in \mathbb{R}$, i.e. $v_{h}$ can be represented as the interpolant of a global constant function. It is therefore immediate to check that the global seminorm $\|\cdot\|_{1, h}$ becomes a norm when restricted to the space with zero boundary conditions as is the case of the linear space $\mathcal{V}_{h, 0}$.

\section{Convergence Analysis}

To analyze the convergence of the method, we need to impose shape-regularity constraints on the sequence of computational meshes, which relies on the mesh regularity assumption taken from $[9,13]$.

Assumption 3.1 (HG). There exist an integer number $\mathcal{N}^{s}$ and a real positive number $\rho_{s}$, whcih are both independent of $h$, and a point $\overline{\mathbf{x}}_{\mathrm{P}}$ inside every polygonal cell $\mathrm{P}$ of every mesh $\Omega_{h}$, such that

( $\mathrm{HG} 1)$ every polygon $\mathrm{P}$ is star-shaped with respect to $\overline{\mathrm{x}}_{\mathrm{P}}$ and has at most $\mathcal{N}^{s}$ edges;

(HG2) every polygon $\mathrm{P}$ admits a triangular decomposition $\mathcal{S}_{h, \mathrm{P}}$ obtained by connecting the vertices of $\partial \mathrm{P}$ to $\overline{\mathrm{x}}_{\mathrm{P}}$;

(HG3) every triangle $\mathrm{T} \in \mathcal{S}_{h}$ is shape-regular in the sense that the ratio of the radius $r_{\mathrm{T}}$ of the inscribed circle to the diameter $h_{\mathrm{T}}$ is bounded from below by $\rho_{s}$ :

$$
0<\rho_{s} \leq \frac{r_{\mathrm{T}}}{h_{\mathrm{T}}} .
$$

Such assumptions allow us to have a great generality in the cell shapes and non-convex cells or degenerate polygons are admissible. Furthermore, all geometric quantities such as edge lengths and cell areas properly scale with $h$ when $h$ tends to zero. A thorough discussion about the implications of such assumptions is detailed in $[9,13]$

Theorem 3.1. Let us assume that $u$, the solution of the variational problem (3) with $g=0$ on $\Gamma$ under assumptions $(H 1)-(H 3)$, belongs to $H^{m+1}(\Omega)$, and let $u^{I} \in \mathcal{V}_{h}$ be its interpolation on the space $\mathcal{V}_{h}$ defined in accordance with 14. Let $u_{h}$ be solution of the MFD problem (4) under assumption (HG) and (S1)-(S2). Then, there exists a positive constant $C$, which depends only on the regularity constants $\mathcal{N}^{s}$ and $\rho_{s}$ and is independent of $h$, such that

$$
\left\|u^{I}-u_{h}\right\|_{1, h} \leq C h^{m}\|u\|_{H^{m+1}(\Omega)} .
$$

The proof of Theorem 3.1 follows the same arguments of the proof of the convergence theorem in [9] that are based on two theoretical tools: the construction of the lifting operator and the stability of the interpolation operator. Once such tools have been established, the proof reported in [9] can be easily adapted to the present case. 
Table 2. Mesh parameters of the meshes used in the numerical experiments.

\begin{tabular}{ccccc}
\hline lev & $N_{P}$ & $N_{F}$ & $N_{V}$ & $h$ \\
\hline 1 & 25 & 120 & 96 & $2.91510^{-1}$ \\
2 & 100 & 440 & 341 & $1.45810^{-1}$ \\
3 & 400 & 1680 & 1281 & $7.28910^{-2}$ \\
4 & 1600 & 6560 & 4961 & $3.64410^{-2}$ \\
5 & 6400 & 25920 & 19521 & $1.82210^{-2}$ \\
6 & 25600 & 103040 & 77441 & $9.11110^{-3}$ \\
\hline
\end{tabular}

\section{Numerical Results}

We solve the diffusion problem (1)-(2) on the domain $\Omega=] 0,1[\times] 0,1[$ and Dirichlet conditions assigned on all the domain boundary $\Gamma$. The right-hand side $f$ and the boundary function $g$ are determined in accordance with the exact solution

$$
u(x, y)=x \sin (2 \pi x) \sin (2 \pi y)+x^{3} y^{2},
$$

and the constant diffusion tensor

$$
\mathrm{K}(x, y)=\left(\begin{array}{cc}
1 & 1 / 4 \\
1 / 4 & 1
\end{array}\right)
$$

We use a sequence of meshes with non-convex elements. The mesh data are reported in Table 2, where the first column lev shows the refinement level, the second, third and fourth column, respectively labeled by $N_{P}, N_{F}$, and $N_{V}$ shows the number of polygons, edges, and vertices, the fifth column labeled by \#dofs shows the number of degrees of freedom, and the sixth column the value of the mesh size parameter $h$. The first and the second mesh of this family are shown in the two plots of Figure 2. The absolute error is measured using the mesh-dependent norm that is defined in (18)-(19):

$$
\mathcal{E}_{a b s}\left(u_{h}\right)=\left\|u-u_{h}\right\|_{1, h},
$$

and the associated relative error is given by

$$
\mathcal{E}_{a b s}\left(u_{h}\right)=\frac{\mathcal{E}_{r e l}\left(u_{h}\right)}{\left\|u^{\mathrm{I}}\right\|_{1, h}},
$$

where the denominator uses the degrees of freedom associated with the exact solution $u_{h}$.

The numerical results are shown in Tables 3, 4, and 5, for the schemes corresponding to $(\alpha, m)=(0,1),(\alpha, m)=(1,2)$, and $(\alpha, m)=(2,3)$, respectively. These tables shows the refinement label in the first columns (labeled by lev), the number of degrees of freedom in the second column (labeled by \#dofs), the absolute error in the third column (labeled by $\mathcal{E}_{a b s}\left(u_{h}\right)$ ), the relative error in the fourth column (labeled by $\mathcal{E}_{\text {rel }}\left(u_{h}\right)$ ), and the rate of convergence in the fifth colummn (labeled by Rate). The rate of convergence is evaluated by comparing the relative errors of each refinement level and of the preceeding one with 

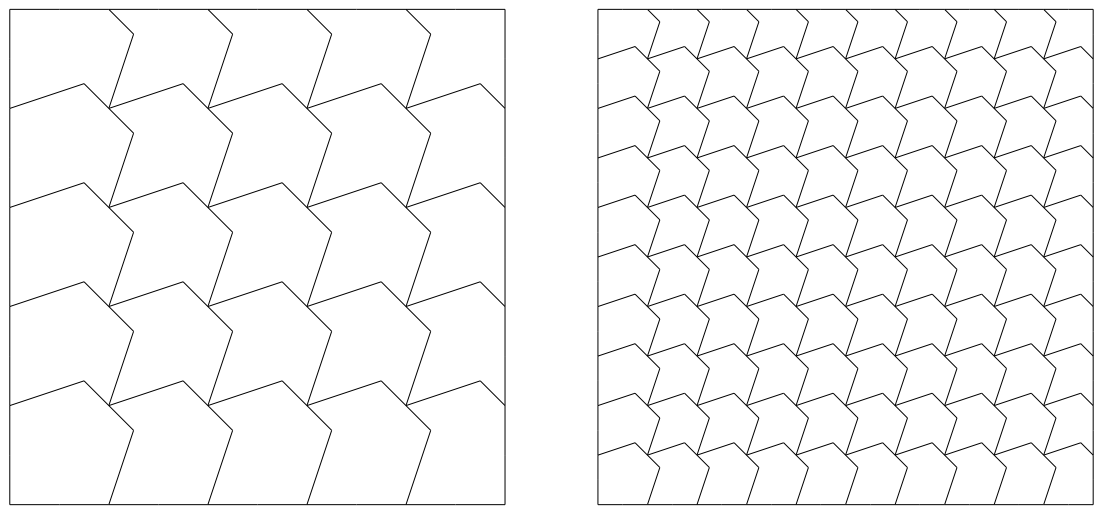

Figure 2. First and second mesh used in the numerical experiments.

respect to the number of degrees of freedom. In accordance with the convergence result of Theorem 3.1, the optimal convergence rate for the approximation errors in (23) and in (24) that is expected for the scheme defined by $(\alpha, m)$ is proportional to $h^{m}$. The numerical results that we obtained are in agreement with the expected behavior of the schemes.

Table 3. Absolute and relative approximation errors in $1 h$-type mesh norm for $(\alpha, m)=$ $(0,1)$; rate is calculated versus the degrees of freedom.

\begin{tabular}{ccccc}
\hline lev & \#dofs & $\mathcal{E}_{a b s}\left(u_{h}\right)$ & $\mathcal{E}_{\text {rel }}\left(u_{h}\right)$ & Rate \\
\hline 1 & 96 & 2.023 & $5.34410^{-1}$ & -- \\
2 & 341 & 1.301 & $3.48110^{-1}$ & 0.676 \\
3 & 1281 & $7.38210^{-1}$ & $2.03110^{-1}$ & 0.813 \\
4 & 4961 & $3.84010^{-1}$ & $1.07610^{-1}$ & 0.938 \\
5 & 19521 & $1.94010^{-1}$ & $5.48110^{-2}$ & 0.985 \\
6 & 77441 & $9.71910^{-2}$ & $2.75710^{-2}$ & 0.997 \\
\hline
\end{tabular}

\section{Conclusions}

In this paper, we presented a new family of mimetic methods on unstructured polygonal meshes for the diffusion problem in primal form. These methods are suitable to the numerical discretization of solutions whoose regularity is $C^{\alpha}(\Omega)$ for any integer $\alpha \geq 0$. The derivation is these mimetic methods is based on a local consistency condition that is exact for polynomials of degree $m=\alpha+1$. The degrees of freedom represents both function values and derivative values of various degree at the mesh vertices and function polynomial moments inside the polygons. A theoretical convergence result is also presented. This result that implies an optimal error estimate in a mesh-dependent norm that mimics the energy norm. Numerical experiments confirmed the convergence rate that is expected from the theory. A more detailed presentation of this family of mimetic methods will be the subject of a forthcoming publication. 
Table 4. Absolute and relative approximation errors in $1 h$-type mesh norm for $(\alpha, m)=$ $(1,2)$; rate is calculated versus the degrees of freedom.

\begin{tabular}{ccccc}
\hline lev & \#dofs & $\mathcal{E}_{\text {abs }}\left(u_{h}\right)$ & $\mathcal{E}_{\text {rel }}\left(u_{h}\right)$ & Rate \\
\hline 1 & 313 & 1.625 & $4.21110^{-1}$ & -- \\
2 & 1123 & $4.14510^{-1}$ & $8.90110^{-2}$ & 2.432 \\
3 & 4243 & $9.71210^{-2}$ & $1.98310^{-2}$ & 2.259 \\
4 & 16483 & $2.38510^{-2}$ & $4.81510^{-3}$ & 2.085 \\
5 & 64963 & $5.94910^{-3}$ & $1.19810^{-3}$ & 2.028 \\
6 & 257923 & $1.48810^{-3}$ & $2.99510^{-4}$ & 2.010 \\
\hline
\end{tabular}

Table 5. Absolute and relative approximation errors in $1 h$-type mesh norm for $(\alpha, m)=$ $(2,3)$; rate is calculated versus the degrees of freedom.

\begin{tabular}{ccccc}
\hline lev & \#dofs & $\mathcal{E}_{a b s}\left(u_{h}\right)$ & $\mathcal{E}_{\text {rel }}\left(u_{h}\right)$ & Rate \\
\hline 1 & 651 & $7.88310^{-1}$ & $1.74410^{-1}$ & -- \\
2 & 2346 & $5.23210^{-2}$ & $1.05410^{-2}$ & 4.376 \\
3 & 8886 & $2.26510^{-3}$ & $4.54310^{-4}$ & 4.722 \\
4 & 34566 & $2.32310^{-4}$ & $4.66310^{-5}$ & 3.352 \\
5 & 136326 & $2.75310^{-5}$ & $5.52810^{-6}$ & 3.107 \\
\hline
\end{tabular}

\section{Acknowledgements}

The work of the second author was partially supported by the National Nuclear Security Administration of the U.S. Department of Energy at Los Alamos National Laboratory under Contract No. DE-AC52-06NA25396 and the DOE Office of Science Advanced Scientific Computing Research (ASCR) Program in Applied Mathematics.

\section{REFERENCES}

[1] Aarnes J. E., Krogstad S., and Lie K.A., "Multiscale mixed/mimetic methods on cornerpoint grids". Computat. Geosci., 12(3):297-315, 2007.

[2] Beirão da Veiga L. "A residual based error estimator for the mimetic finite difference method". Numer. Math., 108(3):387-406, 2008.

[3] Beirão da Veiga L. "A mimetic discretization method for linear elasticity". Math. Mod. and Numer. Anal., 44(2):231-250, 2010.

[4] Beirão da Veiga L., Droniou D., and Manzini G. "A unified approach to handle convection terms in Finite Volumes and Mimetic Discretization Methods for elliptic problems". IMA J. Numer. Anal., 31(4):1357-1401, 2011.

[5] Beirão da Veiga L., Gyrya V., Lipnikov K., and Manzini G.. "Mimetic finite difference method for the Stokes problem on polygonal meshes". J. Comput. Phys., 228(19):72157232, 2009. 
[6] Beirão da Veiga L. and Lipnikov K. "A mimetic discretization of the Stokes problem with selected edge bubbles.” SIAM J. Sci. Comp., 32(2):875-893, 2010.

[7] Beirão da Veiga L., Lipnikov K., and Manzini G. "Convergence analysis of the high-order mimetic finite difference method”. Numer. Math., 113(3):325-356, 2009.

[8] Beirão da Veiga L., Lipnikov K., and Manzini G. "Error analysis for a Mimetic Discretization of the steady Stokes problem on polyhedral meshes". SIAM J. Numer. Anal., 48:1419-1443, 2011.

[9] Beirão da Veiga L., Lipnikov K., and Manzini G. "Arbitrary-order nodal mimetic discretizations of elliptic problems on polygonal meshes". SIAM Journal on Numerical Analysis, 49(5):1737-1760, 2011.

[10] Beirão da Veiga L. and Manzini G. "An a posteriori error estimator for the mimetic finite difference approximation of elliptic problems". Int. J. Numer. Meth. Engrg., 76(11):1696-1723, 2008.

[11] Beirão da Veiga L. and Manzini G. "A higher-order formulation of the mimetic finite difference method". SIAM Journal on Scientific Computing, 31(1):732-760, 2008.

[12] Beirão da Veiga L. and Mora D. "A mimetic discretization of the Reissner-Mindlin plate bending problem". Numer. Math., 117(3):425-462, 2011.

[13] Brezzi F., Buffa A., and Lipnikov K. "Mimetic finite differences for elliptic problems". M2AN Math. Model. Numer. Anal., 43(2):277-295, 2009.

[14] Brezzi F., Lipnikov K., and Shashkov M. "Convergence of the mimetic finite difference method for diffusion problems on polyhedral meshes". SIAM J. Numer. Anal., 43(5):1872-1896, 2005.

[15] Brezzi F., Lipnikov K., and Shashkov M. "Convergence of mimetic finite difference method for diffusion problems on polyhedral meshes with curved faces". Math. Models Methods Appl. Sci., 16(2):275-297, 2006.

[16] Campbell J. and Shashkov M. "A tensor artificial viscosity using a mimetic finite difference algorithm". J. Comput. Phys., 172:739-765, 2001.

[17] Cangiani A., Gardini F., and Manzini G. "Convergence of the mimetic finite difference method for eigenvalue problems in mixed form". Comput. Methods Appl. Mech. Engrg., 200((9-12)):1150-1160, 2010.

[18] Cangiani A. and Manzini G. "Flux reconstruction and pressure post-processing in mimetic finite difference methods". Comput. Methods Appl. Mech. Engrg., 197/9-12:933-945, 2008.

[19] Cangiani A., Manzini G., and Russo A. "Convergence analysis of the mimetic finite difference method for elliptic problems". SIAM J. Numer. Anal., 47(4):2612-2637, 2009.

[20] Gyrya V. and Lipnikov K. "High-order mimetic finite difference method for diffusion problems on polygonal meshes”. J. Comput. Phys., 227:8841-8854, 2008.

[21] Hyman J., Shashkov M., and Steinberg S. "The numerical solution of diffusion problems in strongly heterogeneous non-isotropic materials". J. Comput. Phys., 132:130-148, 1997.

[22] Lipnikov K., Manzini G., Brezzi F., and Buffa A. "The mimetic finite difference method for 3D magnetostatics fields problems". J. Comput. Phys., 230(2):305-328, 2011. 
[23] Lipnikov K., Manzini G., and Svyatskiy D. "Analysis of the monotonicity conditions in the mimetic finite difference method for elliptic problems". J. Comput. Phys., 230(7):2620 - 2642, 2011.

[24] Lipnikov K., Morel J., and Shashkov M.. "Mimetic finite difference methods for diffusion equations on non-orthogonal non-conformal meshes”. J. Comput. Phys., 199, 2004.

[25] Lipnikov K., Moulton J. D., and Svyatskiy D. "A Multilevel Multiscale Mimetic ( $\left.{ }^{3}\right)$ method for two-phase flows in porous media”. J. Comp. Phys., 227:6727-6753, 2008.

[26] Morel J., Roberts R., and Shashkov M. "A local support-operators diffusion discretization scheme for quadrilateral $r-z$ meshes". J. Comput. Phys., 144:17-51, 1998.

[27] Shashkov M. and Steinberg S. "Solving diffusion equations with rough coefficients in rough grids". Journal of Computational Physics, 129(2):383 - 405, 1996. 